\title{
Graphical Visualization of Human Exploration Capabilities
}

\author{
Erica M. Rodgers, Julie Williams-Byrd†, Dale C. Arney*, \\ Matthew A. Simon; $\ddagger$ and Phillip A. Williams ${ }^{\dagger}$ \\ NASA Langley Research Center, Hampton, VA 23681 \\ Christopher Barsoum ${ }^{\S}$ \\ University of Leicester, Leicester, United Kingdom \\ Tyler Cowan ${ }^{\S}$ \\ Columbia University, New York, NY 10027 \\ Kevin T. Larman ${ }^{\ddagger}$ \\ Analytical Mechanics Associates Inc., Hampton, VA 23666 \\ Jason Hay and Alex Burg" \\ The Tauri Group, Alexandria, VA 22310
}

\begin{abstract}
NASA's pioneering space strategy will require advanced capabilities to expand the boundaries of human exploration on the Journey to Mars (J2M). The Evolvable Mars Campaign (EMC) architecture serves as a framework to identify critical capabilities that need to be developed and tested in order to enable a range of human exploration destinations and missions. Agency-wide System Maturation Teams (SMT) are responsible for the maturation of these critical exploration capabilities and help formulate, guide and resolve performance gaps associated with the EMC-identified capabilities. Systems Capability Organization Reporting Engine boards (SCOREboards) were developed to integrate the SMT data sets into cohesive human exploration capability stories that can be used to promote dialog and communicate NASA's exploration investments. Each SCOREboard provides a graphical visualization of SMT capability development needs that enable exploration missions, and presents a comprehensive overview of data that outlines a roadmap of system maturation needs critical for the J2M. SCOREboards are generated by a computer program that extracts data from a main repository, sorts the data based on a tiered data reduction structure, and then plots the data according to specified user inputs. The ability to sort and plot varying data categories provides the flexibility to present specific SCOREboard capability roadmaps based on customer requests. This paper presents the development of the SCOREboard computer program and shows multiple complementary, yet different datasets through a unified format designed to facilitate comparison between datasets. Example SCOREboard capability roadmaps are presented followed by a discussion of how the roadmaps are used to: 1) communicate capability developments and readiness of systems for future missions, and 2) influence the definition of NASA's human exploration investment portfolio through capability-driven processes. The paper concludes with a description of planned future work to modify the computer program to include additional data and of alternate capability roadmap formats currently under consideration.
\end{abstract}

*Aerospace Engineer, Space Mission Analysis Branch, 1 North Dryden Street, Hampton, VA, 23681, AIAA Member

$\dagger$ Aerospace Engineer, Space Mission Analysis Branch, 1 North Dryden Street, Hampton, VA, 23681

$¥$ Aerospace Engineer, Space Mission Analysis Branch, 1 North Dryden Street, Hampton, VA, 23681, AIAA Senior Member

$\S$ NASA Intern, Space Mission Analysis Branch, 1 North Dryden Street, Hampton, VA, 23681

TSenior Technology Analyst, The Tauri Group, 6363 Walker Ln Ste 300, Alexandria, VA 22310

" Technology Analyst, The Tauri Group, 6363 Walker Ln Ste 300, Alexandria, VA 22310 


\section{Nomenclature}

\begin{tabular}{|c|c|}
\hline $\mathrm{AMO}$ & Autonomous Mission Operations \\
\hline $\mathrm{ARCM}$ & Asteroid Redirect Crewed Mission \\
\hline ARRM & Asteroid Redirect Robotic Mission \\
\hline $\mathrm{CDF}$ & Capability-Driven Framework \\
\hline $\mathrm{CHP}$ & Crew Health \& Protection and Radiation \\
\hline Comm/Nav & Communication and Navigation \\
\hline ECLSS & Environmental Control and Life Support Systems \\
\hline EDL & Entry, Decent and Landing \\
\hline EM-1 & Exploration Mission 1 \\
\hline EM-2 & Exploration Mission 2 \\
\hline EMC & Evolvable Mars Campaign \\
\hline EVA & Extra-vehicle Activity \\
\hline FY & fiscal year \\
\hline GUI & Graphical User Interface \\
\hline HEOMD & Human Exploration and Operations Mission Directorate \\
\hline $\mathrm{ICH}$ & initial cislunar habitation \\
\hline ISS & International Space Station \\
\hline ISRU & In-Situ Resource Utilization \\
\hline $\mathrm{J} 2 \mathrm{M}$ & Journey to Mars \\
\hline LEO & low-Earth orbit \\
\hline Power & Power and Energy Storage \\
\hline Robotics & Human-Robotic Mission Operations \\
\hline $\mathrm{RPM}$ & Resource Prospector Mission \\
\hline SCOREboard & Systems Capability Organization Reporting Engine board \\
\hline SEP & Solar Electric Propulsion \\
\hline SLS & Space Launch System \\
\hline $\mathrm{SME}$ & Subject Matter Expert \\
\hline SMMP & Structures, Mechanisms, Materials and Processes \\
\hline SMT & System Maturation Team \\
\hline SOA & state-of-the-art \\
\hline TBD & to-be-determined \\
\hline
\end{tabular}

\section{Introduction}

NASA's pioneering space strategy will utilize advanced capabilities to expand the boundaries of human exploration on the Journey to Mars (J2M). These capabilities will allow humans to work, learn, operate, live, and thrive safety beyond the Earth for extended periods of time. This pioneering approach follows a set of key strategic principles, which include sustained investments for technologies and capabilities that will address challenges of future missions and incremental buildup of capabilities for more complex missions over time. ${ }^{1}$

\section{A. Evolvable Mars Campaign}

The Evolvable Mars Campaign (EMC) is an ongoing Mars mission planning effort featuring a series of trade analyses to define the capabilities and elements needed to enable NASA's J2M, starting from today's capabilities and leading to a sustainable human presence on the surface of Mars. The Human Exploration and Operations Mission Directorate (HEOMD) leads the campaign, with participation across nine of the ten NASA centers and close coordination with other architectural analysis groups, the Science and Space Technology Mission Directorates, and the Offices of the Chief Scientist and the Chief Technologist. The 
EMC routinely receives inputs from external organizations as well, including international partners, industry, academia, and NASA advisory groups.

The EMC identifies a set of operational capabilities and architectural trades required to sustainably expand human presence from low-Earth orbit (LEO) into deep space. The capability-driven EMC integrates science missions, robotic precursors, system maturation and risk reduction pathfinders, and a sustainable cadence of cargo and crewed missions that lead to an extended human presence on the surface of Mars.

Several scenarios have been considered for a human mission to the Martian surface. Of these, only one spans all Mars vicinity destinations. The "Mars vicinity and Phobos, followed by a mission to Mars surface" scenario represents an ambitious campaign that leverages most of the capabilities and potential tradeoffs described in the EMC. This particular campaign acts as a point of comparison for future trade assessments and serves as the baseline reference campaign for the EMC. This baseline reference scenario is then used to evaluate capabilities, schedules, risks, challenges, and mitigation strategies.

\section{B. Exploration Phases}

The EMC is actively defining tradeoffs to enable a sustainable exploration endeavor to expand human presence from LEO into the solar system, specifically to the surface of Mars. Figure 1 depicts the EMC, which integrates science missions, robotic precursor missions, capability pathfinder missions, and a sustained cadence of crewed missions and activities that lead to humans on the surface of Mars. Although an order of progression is indicated, potential paths to Mars do not necessarily have to explore the same interim destinations, nor accomplish identical objectives at those destinations. The EMC is designed to explore a range of capabilities addressing the challenges associated with potential paths to destinations. The intent of the EMC is to inform capability development and an assessment of options without prescribing a path to Mars. Beyond the bounding date for Mars vicinity and Mars surface missions, specific dates have not been assigned to the missions associated with the EMC. Work has begun to explore a range of assumed dates to assess viability from a technical and programmatic perspective.

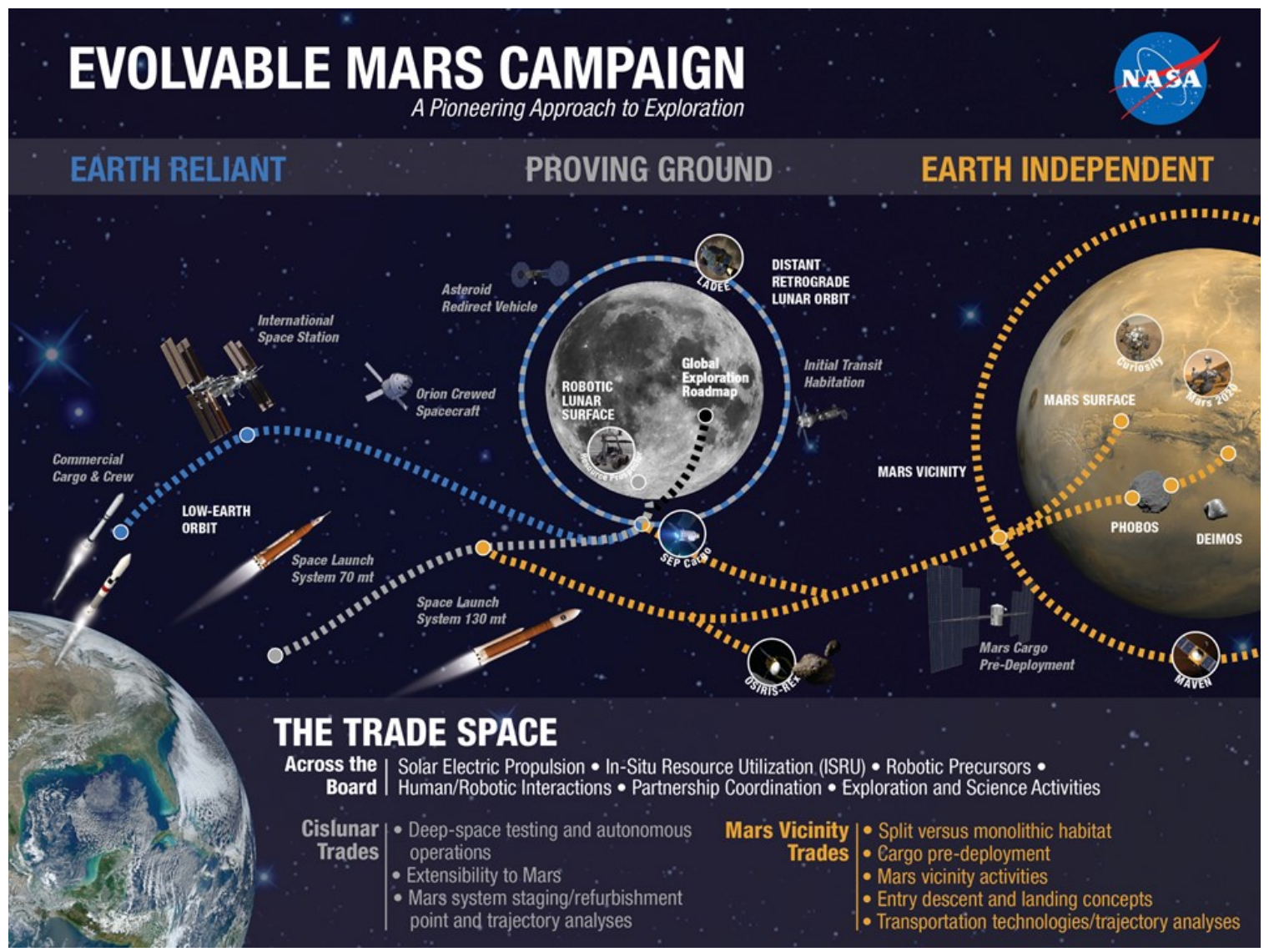

Figure 1. The Evolvable Mars Campaign Architecture Analysis Trade Space. 
Following the J2M strategy, the EMC is organized in four phases. It begins with Earth Reliant Phase 0 missions to expand the knowledge of operations in space, transitions to missions in cislunar space (e.g., lunar orbit) in Proving Ground Phase 1 and 2 for testing and certification of required capabilities, and ultimately enables Earth Independent missions and long-duration stays on the Martian surface in Phase 3 and beyond (Figure 2).

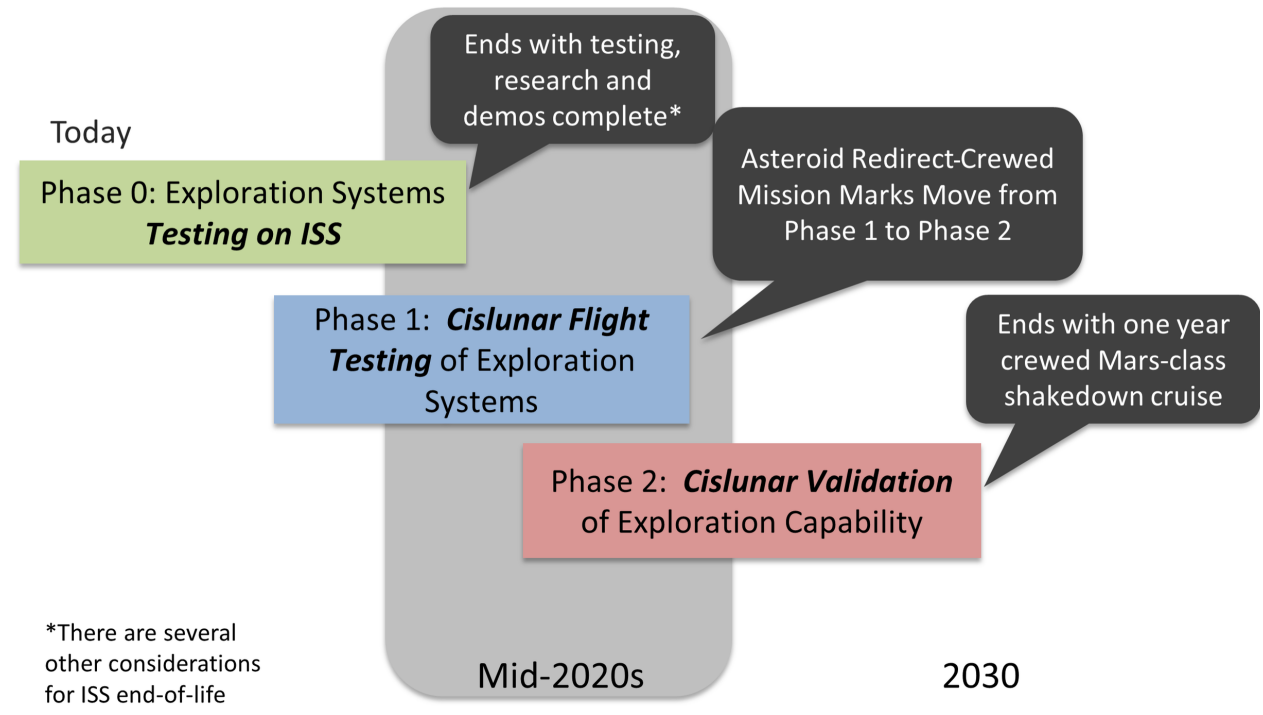

Figure 2. Phase 0 - Phase 2 framework for transitioning from Earth Reliant exploration activities on ISS to Proving Ground missions in cislunar space.

\section{B.1. Earth Reliant/Phase 0}

Earth Reliant exploration activities, also referred to as Phase 0, address whether humans can live and operate for approximately 1,000 days in microgravity. Phase 0 leverages investments in the ISS to advance exploration systems and long duration human testing. NASA will use the ISS to conduct microgravity testing of Mars-forward capabilities, ranging from individual components to Mars-ready environmental control and life support systems. Increasing the duration of crewed expeditions beyond the typical 180 day ISS mission will help to better understand the effectiveness of counter measures required for the nominal Mars mission of approximately 1,000-days in deep space. Exploration capability testing on ISS will support activities in the Proving Ground and will validate the capabilities that will enable future Mars human missions. Development of this knowledge is essential before ISS can be transitioned to commercial use sometime between 2024 and 2028.

\section{B.2. Proving Ground: Phase 1 \& Phase 2}

The next phases of human exploration activity focus on achieving key Proving Ground Phase 1 and 2 objectives, before transitioning to Earth Independent exploration. Key elements for validation include the Space Launch System (SLS) and Orion; advanced solar electric propulsion (SEP), as demonstrated through the Asteroid Redirect Mission; the initial cislunar habitation facility enabling human presence in deep space; and a Mars transit habitat protoflight vehicle enabling long duration habitation testing. The Proving Ground timeframe also requires investments in large-scale entry, descent, and landing systems, landers, and longduration Mars surface systems because of the 10-15 year lead time to develop the required capabilities.

Proving Ground missions also allow NASA to test materials and systems in the deep space environment, outside from Earth's magnetic field. While enabling the incremental build-up of capabilities and infrastructure. In Phase 1 of the Proving Ground, SLS/Orion will be launching once per year beginning in 2021 and while the sequence of activities are still being determined, key missions and capability demonstrations have been identified through the mid-2020s to ensure progress toward Earth Independence. These Phase 1 demonstrations include the first integrated test of SLS and Orion in 2018 with Exploration Mission 1 (EM-1); the launch of the asteroid robotic redirect mission (ARRM) and SEP demonstration in 2020; the first crewed Proving Ground mission on EM-2 and launch of an initial cislunar habitation (ICH) facility; and 
subsequent EMs will transition into Proving Ground Phase 2 missions that will encompass the first crewed mission to the redirected asteroid along with long-duration testing and operations for crew and systems. Figure 3 highlights notional human and robotic missions within the exploration phases framework.

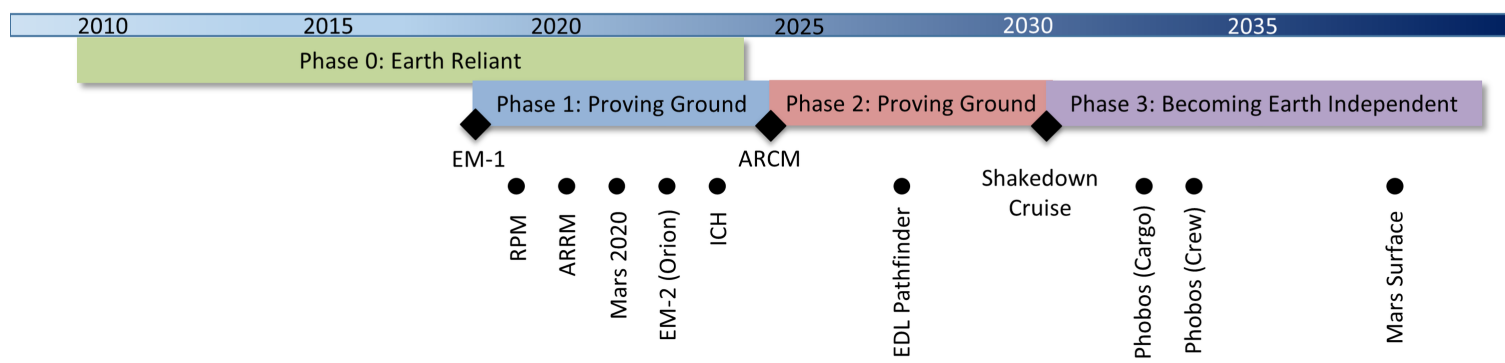

Figure 3. Notional human and robotic mission launch dates within the exploration phases framework. $\mathrm{RPM}=\mathrm{Resource}$ Prospector Mission and ARRM = Asteroid Redirect Robotic Mission.

Phase 2 Proving Ground missions, beginning with the Asteroid Redirect Crewed Mission (ARCM), will not only demonstrate the ability to live and work beyond LEO, but they will accomplish a suite of Proving Ground Phase 2 objectives and validate key operational capabilities required to becoming Earth Independent in the 2030s. In Phase 2, we will also learn how to reduce logistics, reuse packaging, and recycle materials - knowledge essential for success as we extend our missions across the solar system. The Mars protoflight habitat would be used to test habitation capabilities required for Mars missions that are not addressed by the ISS or the initial small crew-tended habitat. That same habitat could then be used for an initial crewed mission to the Mars vicinity after an extended "shakedown" period with crew (Figure 3).

\section{B.3. Becoming Earth Independent - Phase 3 and Beyond}

The next stage of exploration activity focuses on exploration of the Mars vicinity, including Phobos, Deimos, Mars orbit, and the Martian surface. Crewed exploration missions in this phase exercise the Mars transportation system, potentially explore the moons, and facilitate teleoperations of Mars surface systems for reconnaissance and infrastructure deployment. The Mars surface missions continue the transition from exploration to pioneering, with extended human presence in the Mars vicinity and eventually on the surface of Mars becoming increasingly less dependent on resources from Earth (Figure 3).

In becoming Earth Independent, humans will increasingly become less dependent on assistance from mission control and increase their autonomous operating capability. This begins with mission focus on human access to the Mars system, without requiring surface access. This could include a long duration crewed mission to Phobos (with Deimos as a strong alternative), followed by a crewed mission to the surface of Mars. This approach delays the investments required for Mars surface access (i.e., entry, descent, and landing, surface habitation, surface exploration systems such as rovers and surface power, etc.), to spread out development costs, while still allowing humans to explore the Mars system.

The latter part of becoming Earth Independent addresses access to the Martian surface and whether humans can break the supply chain with Earth to enable a sustained presence on the surface of Mars. This includes Mars surface access and development of Mars infrastructure. Initial missions include crew exploration of the Mars surface and emplacement of surface infrastructure. Subsequent missions return to the same landing site as the first mission, leveraging the existing infrastructure, while deploying and testing systems that reduce dependence on a supply chain from Earth. The eventual goal in this phase is that only the crew needs to be transported to Mars, with all logistics required for their stay extracted from local resources. Each of these phases requires a significant amount of investment before moving on to the next phase, as well as validation of exploration systems and operations that will inform how the next phase is implemented.

\section{Capability-Driven Framework}

NASA codified a capability-driven approach that provides flexible development and maturation of common capabilities necessary to enable the exploration phases of the EMC within the J2M strategy. This capability-driven framework (CDF) defines evolving capabilities that would enable increasingly complex human exploration missions over time. The CDF also provides increased flexibility, greater cost effectiveness, 
and sustainability. The CDF describes an exploration path that follows incremental steps to build, test, refine, and qualify critical capabilities that will lead to affordable flight elements and deep space capability, eventually enabling crewed planetary exploration to destinations beyond the Earth-moon system, such as the surface of Mars. ${ }^{2}$

Since 2013, NASA's HEOMD has engaged in an Agency-wide process to define and refine human exploration capabilities and associated gaps in performance. NASA identified 12 common capabilities ranging from environmental control and life support systems to robotics, and established Agencywide teams or working groups comprised of subject matter experts (SMEs) that are responsible for the maturation of these exploration capabilities. These teams, called the System Maturation Teams (SMTs) help formulate, guide and resolve performance gaps associated with the identified exploration capabilities. $^{2}$ Table 1 lists the SMTs, as well as the crosscutting discipline teams.

The process NASA uses to establish, integrate, and manage the SMT exploration capability data is iterative. NASA relies on the HEOMD's SMT Integration Team within the Advanced Exploration Systems Division to coordinate and facilitate the SMT process. The SMT Integration Team conducts regular reviews and coordination meetings among the SMTs and has developed a number of methods to help the Agency communicate and implement capability-driven processes. ${ }^{2}$

Clearly communicating the SMT human exploration capability data can be challenging because there are many aspects to the data. Often one aspect alone is not sufficient in communicating relevant information due to mutually dependent data.

Table 1. SMT capability areas and the crosscutting discipline teams

System Maturation Team

$$
\text { Autonomous Mission Operations (AMO) }
$$

Communication and Navigation (Comm/Nav)

Crew Health \& Protection and Radiation (CHP)

Environmental Control \& Life Support Systems

and Environmental Monitoring (ECLSS-EM)

Entry, Descent and Landing (EDL)

Extra-vehicle Activity (EVA)

Fire Safety

Human-Robotic Mission Operations (Robotics)

In-Situ Resource Utilization (ISRU)

Power and Energy Storage (Power)

Propulsion

Thermal (including cryo)

Disclipline Team - Crosscutting

Avionics
Structures, Mechanisms, Materials,
and Processess (SMMP)

Therefore, multiple aspects of the data must be presented at the same time in order to understand how these aspects come together to describe comprehensive capability developments. These include development timelines mapped to notional missions in order to understand the readiness of systems for these future missions, as well as funding status and testing location (ISS or cislunar) descriptions in order to determine how these capability developments influence the definition of NASA's human exploration investment portfolio. Integrating these data to communicate comprehensive capability developments requires an improved visualization method.

The Systems Capability Organization Reporting Engine board (SCOREboard) was developed to integrate the 12 sometime disparate SMT data sets into a cohesive human exploration capability story that can be used to communicate NASA's exploration investments within the capability-driven framework. The SCOREboards are graphical visualizations of SMT capability needs that enable the EMC architecture and notional missions. The SCOREboards present an overview of data that provide a roadmap of system maturation critical for the J2M. The SCOREboards are generated by a computer program that extracts data from a main repository, sorts the data based on a tiered data reduction structure, and then plots the data according to specified user inputs. The ability to sort and plot varying data categories provides the flexibility to present specific SCOREboard capability roadmaps based on customer requests.

This paper begins in Section 2 with a description of the SMT data and data collection process. Section 3 describes the development of the SCOREboard computer program and how multiple complimentary, yet different datasets are presented though a unified format designed to facilitate comparison between datasets. Example SCOREboard capability roadmaps are presented in Section 4 followed by a discussion of how the roadmaps are used to: 1) communicate capability developments and readiness of systems for future missions, and 2) influence the definition of NASA's human exploration investment portfolio through capability-driven 
processes. The paper concludes in Section 5 with a description of planned future work to modify the computer program to include additional data and of alternate capability roadmap formats currently under consideration.

\section{System Maturation Team Data}

The SMT data describe gaps in system capability where performance beyond state-of-the-art (SOA) is required for human exploration. The SMTs identified system performance parameters within the context of capability areas where advancements above SOA are necessary. These capability advancements are highlighted in NASA's J2M plan. ${ }^{3}$ Subsequent data from the SMTs and crosscutting discipline teams were provided regarding necessary activities or tasks required to close the identified performance gaps, along with SME assessments of task development durations, task testing location, and capability mission need within the EMC architecture framework. Figure 4 illustrates how multiple data sets come together to make up the complete collection of SMT data. Figure 4 also highlights the full complement of data products resulting from human exploration capability development analyses of the SMT data.

The SMT Integration Team collects and stores the SMT capability data in a central repository using tiered organization to provide a hierarchical structure to the data (Figure 5):

Tier 1 Capability areas where performance improvements in one or more functions is required for human exploration missions

Tier 2 Specific gaps in capability performance highlighting the difference between SOA and required performance

Tier 3 Gap data including mission criticality and mission need of each gap, and the associated tasks required to close the performance gaps

Tier 4 Gap-closing task data including task development durations, task testing locations, and task funding status

The SMT dataset is also complex because each of the 12 systems is complementary, yet different in its level of available detail or granularity. The SMT data are grouped into three categories with respect to their granularity: high, mid, and low (Figure 6). Systems relating to CHP, ECLSS-EM, EVA, Fire Safety, Power, and Thermal have experienced long-term use in a relevant environment aboard the ISS. Additionally, the operational use of these systems is generally well defined for the EMC architecture and notional missions. The combination of these factors allows the SMT to provide very detailed capability data at all tiers in the hierarchy structure (Figure 5); therefore, the CHP, ECLSS-EM, EVA, Fire Safety, Power, and Thermal SMT data comprise the high granularity data group.

System data from the AMO, Comm/Nav, and Robotics SMTs make up the mid granularity data group. Similar to the high granularity data group, these systems have long-term use in a relevant environment (ISS) from which lessons learned can be applied toward Mars missions. However, the operational use of these systems is not well defined for the EMC architecture and notional missions. As a result, the AMO, Comm/Nav, and Robotics SMTs cannot always provide comparable details within their capability data.

The low granularity data group accounts for systems that lack heritage in relevant environments, such as EDL, ISRU, and Propulsion. These systems need more operational time in relevant environments in order to fully understand the advancements above SOA that are required for the EMC architecture and notional missions. Additionally, capability data regarding these systems lack specificity because a more robust EMC operational plan is required. Capability data from EDL, ISRU, and Propulsion are generally more broad and less specific at all levels in the hierarchy structure because of these reasons.

The Avionics and SMMP discipline teams provide system data that are crosscutting to all 12 SMTs. Avionics and SMMP performance gaps generally map to existing SMT gaps; therefore, the discipline data fall in the granularity category appropriate to whichever SMT the designated performance gap pertains.

The SMT capability data presented in this paper are notional and enable the baseline reference campaign using the combination chemical propulsion and solar electric propulsion transportation architecture. These notional data represent a snapshot in time corresponding to the end of 2015 fiscal year (FY). For more information on the EMC campaign and EMC transportation architecture options please refer to Goodliff et al.2016, Merrill et al., 2016, and Percy et al., 2016. ${ }^{4-6}$ 


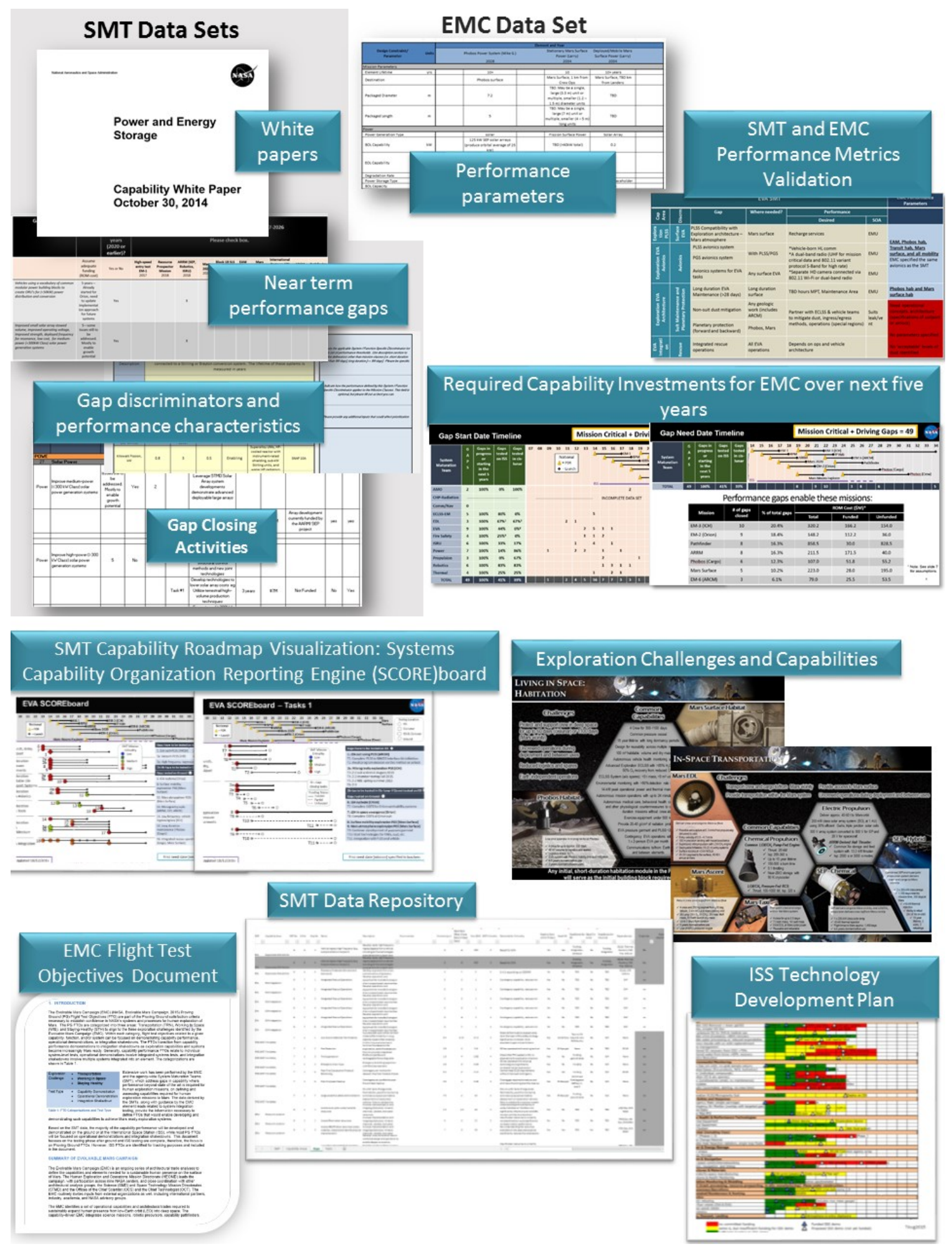

Figure 4. The complete collection of SMT data is comprised of several datasets compiled together. These data are used to analyze human exploration capability developments resulting in numerous products.

\section{SCOREboard Visualizations}

The complexity of the SMT data regarding the varying level of details in each tier provides a challenge when analyzing the system data because the granularity is not consistent throughout the SMT dataset. The solution is to establish a data baseline from which all analysis originates. The baseline data points are used 


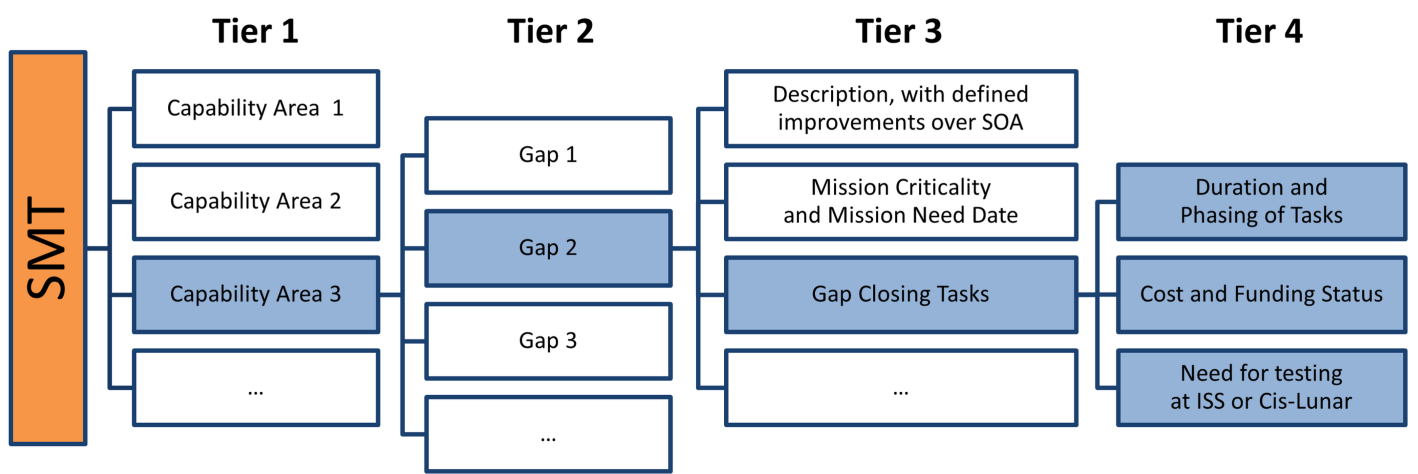

Figure 5. All SMT data are stored in a central repository using this tiered organization to provide a hierarchical structure. The blue highlighted boxes provide an example of how the information flows from left to right going from first order data in Tier 1 to more descriptive data in Tier 4.

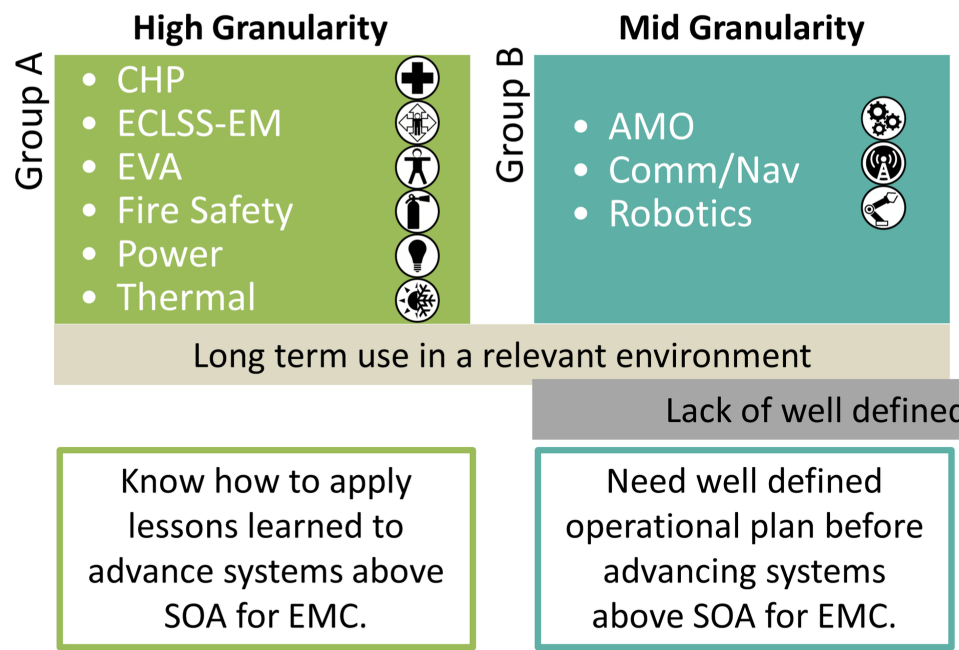

\section{Challenge: Level of detail not consistent throughout SMT data. Solution: Baseline the data at the same level for data analysis.}

Figure 6. The SMT data are grouped into three categories (high, mid, and low) with respect to their data granularity at all tiers within the hierarchy structure.

to determine differences between key aspects of each system maturation roadmap within the framework of the EMC architecture.

The baseline data points come from each of the four data hierarchy tiers and are consistent with the level of detail in the low granularity data group. The baseline data are:

- capability area titles = title only of each SMT-identified capability area

- performance gap titles = title only of each SMT-identified performance gap

- gap-closing task titles = title only of each SMT-identified gap-closing task

- mission criticality numerical scores = single numerical score of 1-5 (lowest-highest) indicating SMT assessment mission criticality

- mission need dates = notional launch date of EMC missions for which an advanced capability is required 
- task durations $=$ numerical length of time in years to complete an identified gap-closing task

- testing location = identified testing location (ground, ISS, or cislunar) for each gap-closing task

- task funding status = FY 2015 funding status (fully funded, partially funded, or unfunded) of each gap-closing task

These data are used to construct visualizations that highlight the maturation of human exploration capabilities in order to compare similarities and differences among each system. These visualizations are called SCOREboards.

SCOREboards are a graphical visualization of SMT capability data that enable the EMC architecture and missions, and are generated by a software computer code. The SCOREboard code uses the baseline SMT data as input, which is then organized into Python data structures. Logic statements are employed to arrange the baseline data for plotting and visualization. The code has the capability to plot the graphics to a static display directly or through the use of the graphical user interface (GUI) shown in Figure 7. The SCOREboard program allows for flexibility so that once the data are arranged into a structure, the user can efficiently plot any data that is requested for visualization whether by choosing different selections on the GUI or by adding additional plotting lines of code. This flexibility allows frequent data updates to be quickly implemented, re-plotted and analyzed; thus, making the SCOREboards an important tool used to communicate timely information regarding SMT capability developments to stakeholders.

\section{A. Data Sorting}

Three sorting categories are used to structure the ways in which the data can be dynamically reduced in a customizable fashion: primary sort, secondary sort, and table sort. Each sorting category is used to determine the data reduction and type of visualization based on user input. Table 2 lists the sorting options for all three categories.

Table 2. Data sorting options used to determine the data reduction and visualization based on user selected baseline data input.

\begin{tabular}{lll} 
Primary Sort & Secondary Sort & Table Sort \\
\hline \hline SMT & Capability Area & SMT \\
Testing Location & Testing Location & Testing Location \\
First Mission & First Mission & First Mission \\
Mission & Mission & \\
& SMT & \\
\hline
\end{tabular}

The primary sort defines the first level of data reduction, which clarifies the focus of the SCOREboard. The secondary sort refers to reducing the data within the requested primary sort. The secondary sort groups together data from within the primary sorting category and defines the order in which the data are visually displayed. The table sort defines the ordering within the descriptive table. Figure 8 shows a SCOREboard template and highlights where the three sorting options are visualized within the template.

\section{B. SCOREboard Template}

The SCOREboard template is organized according to user selected data sorting options, then the rest of the baseline data are added to the make the complete template. A notional mission launch timeline based on Figure 3 is displayed, which highlights key missions spanning the Earth Reliant, Proving Ground, and Earth Independent phases. Performance gap development timelines are plotted along with shapes that indicate the testing location of the gap (ISS, cislunar space, ISS and cislunar, or ground). Each shape is colored to identify the mission criticality of that gap relative to the notional missions. A descriptive table is displayed, which lists the names of all of the gaps corresponding to the numbers plotted next to each gap. The top of Figure 8 displays the SCOREboard template at the performance gap level. 

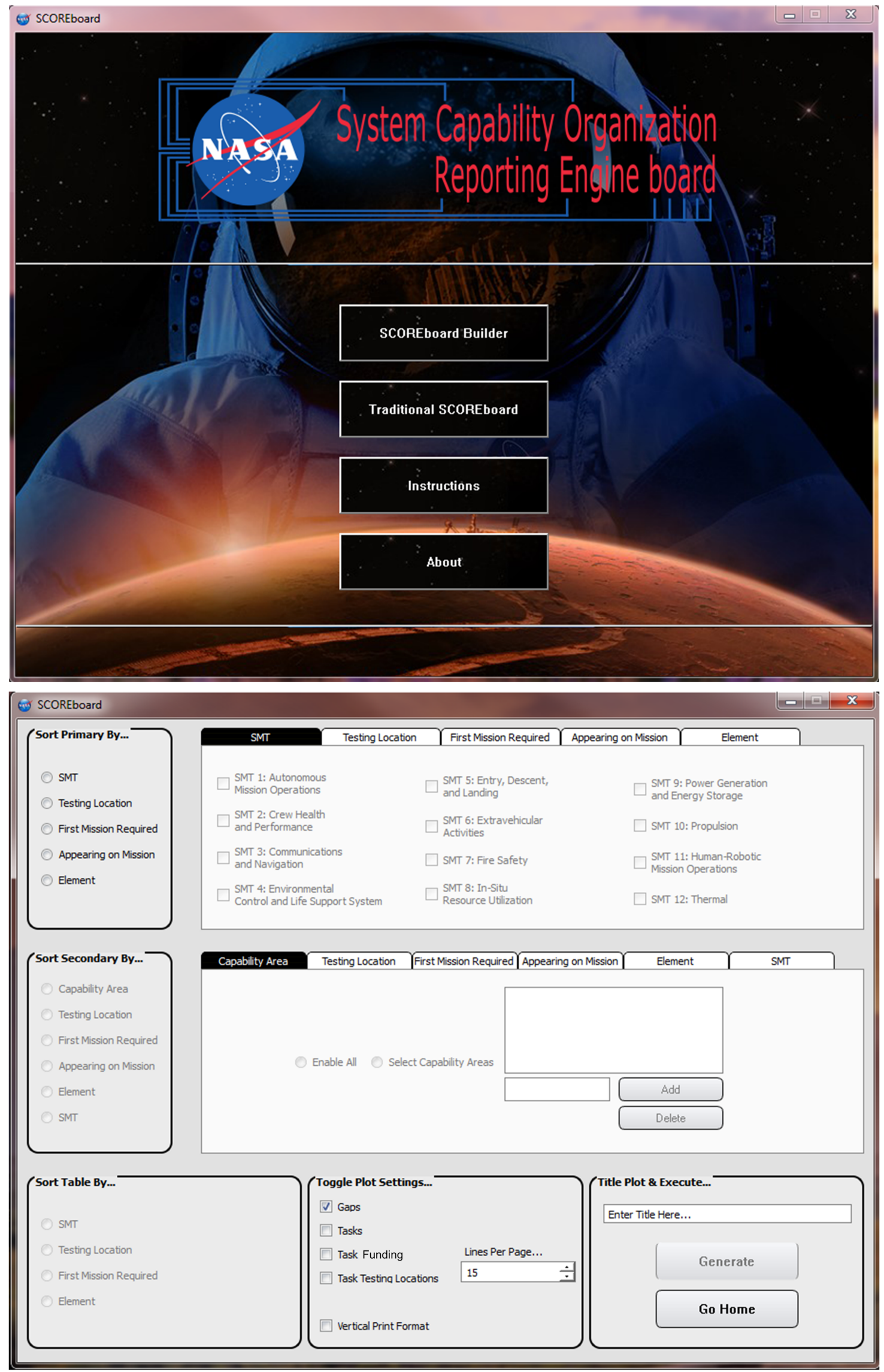

Figure 7. The SCOREboard GUI facilitates a quick turnaround of data products to address stakeholder needs. The BOTTOM image represents the SCOREboard Builder, which allows the user to specify the data sorting options.

\section{1 of 18}




\section{PRIMARY SORT}
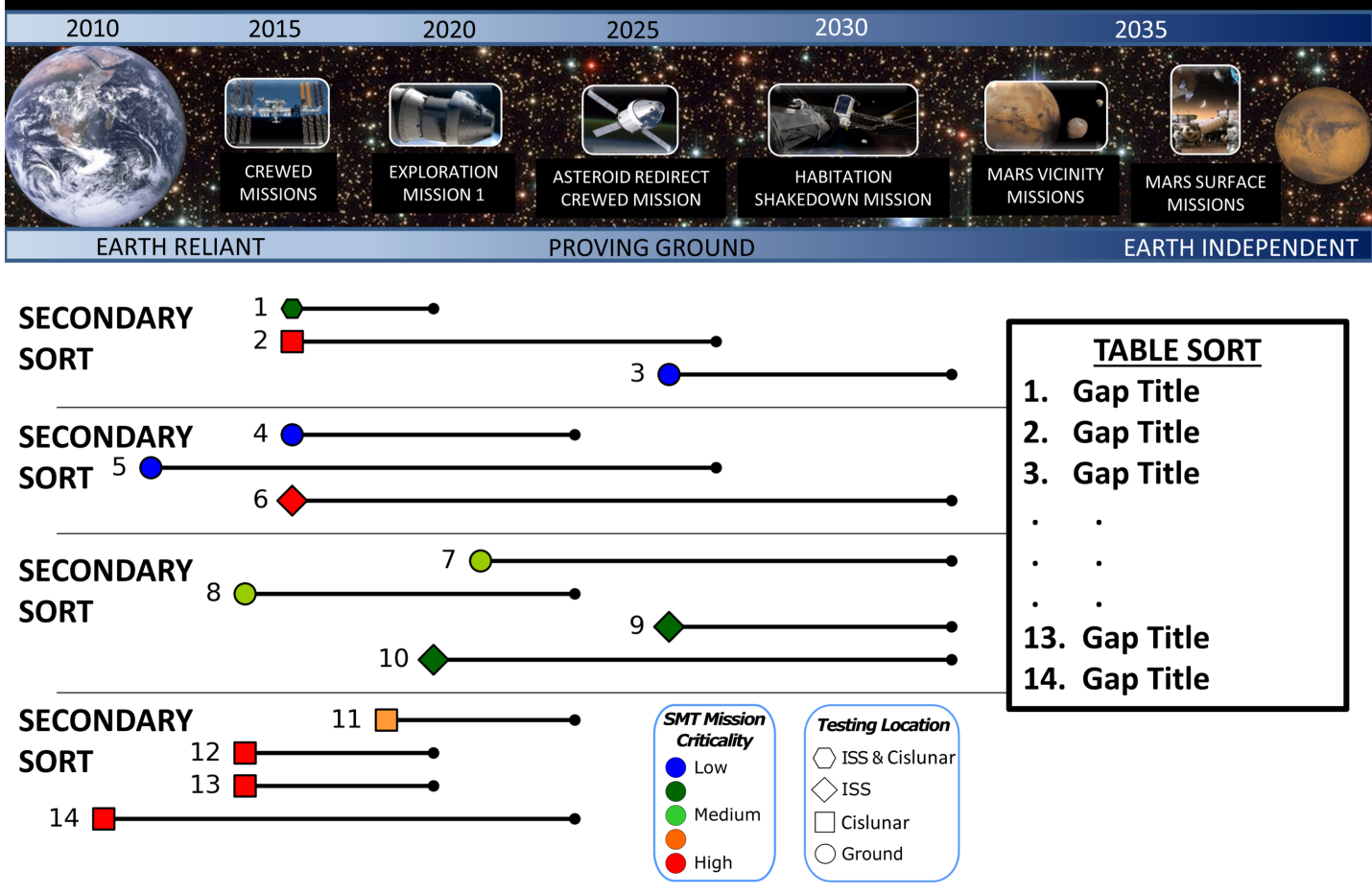

\section{PRIMARY SORT}

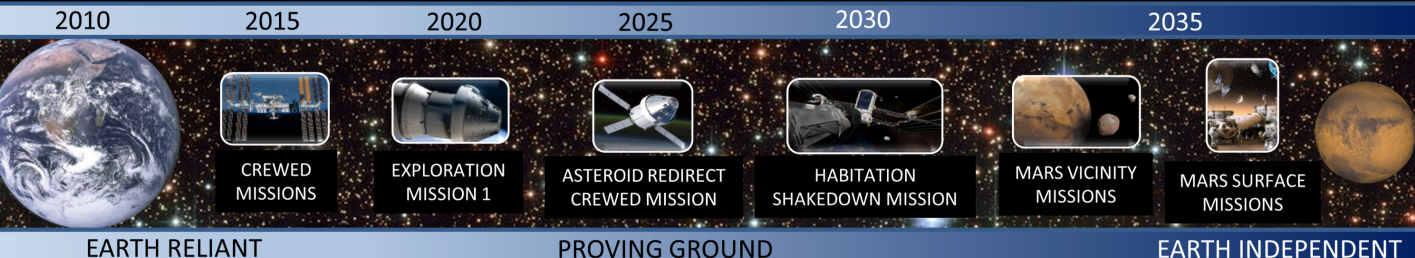

EARTH RELIANT

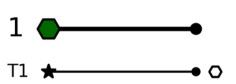

SECONDARY SORT

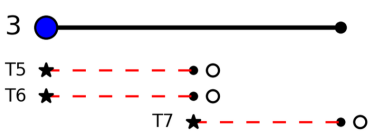

4$$
8 \text { T9 }
$$$$
\text { T9 } \underset{\mathrm{T} 10 \star-}{\star} \mathrm{*}
$$

SECONDARY

$\star-\bullet 0$ SORT

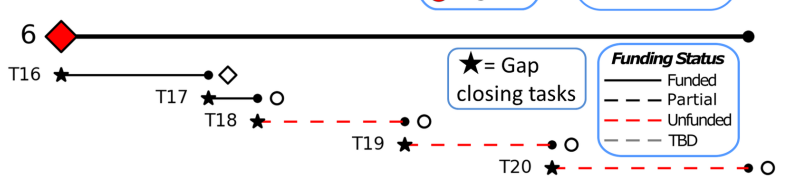

TABLE SORT

1. Gap Title

T1. Task Title

3. Gap Title

T5. Task Title

4. Gap Title

T8. Task Title

6. Gap Title

T16. Task Title

Figure 8. The SCOREboard templates highlight the three data sorting categories: primary sort, secondary sort, and table sort. Any option listed in Table 2 can be selected for each sorting category. The TOP template is used to display data at the performance gap level, while the BOTTOM template is used to display data at the gap-closing task level. 
The SCOREboard template is also used to display data at the gap-closing task level (bottom of Figure 8). The SCOREboard task template includes gap-closing task development timelines plotted underneath the corresponding parent gaps, shapes plotted at the end of each task timeline to indicate the testing location of each task, and varying line styles and line colors to represent the funding status of each task (funded, partially funded, unfunded, or to-be-determined (TBD)). The task titles are displayed within the descriptive table along with their corresponding parent gap title.

\section{SCOREboard Computer Code}

The SCOREboard code version 3.0 was written in the Python computer programming language, using the Python 2.7.11 release. Version 3.0 of the SCOREboard code was designed as a dynamic and nonlinear computer program, capable of expanding on versions 1.0 and 2.0 of the SCOREboard code by enabling the user to be able to more efficiently organize and visualize data as per request.

The SCOREboard source code has two main sections, a functional code and a graphical code. The functional code is the computational portion of the program, which uses a collection of user inputs to output a SCOREboard visualization, and the graphical code is based on a GUI, which operates as a control board for the functional portion of the code, sending in a series of user selected on and off switches for the functional code to interpret. The GUI enhances the usability of the code, guides the user into successful operation of the code, and speeds up the user experience when interacting with the code.

\section{C.1. Functional Code}

The functional code is divided into five main sections based on the following functions: data import, control board, data reduction, graphing, and data export. The description of each section is as follows:

\section{Data Import}

The data import section imports data into the code utilizing the xlrd Python library to extract data from the Excel workbook based SMT repository. Each worksheet within the workbook is converted into a Python dictionary data structure, using the worksheet column titles as dictionary keys. Subsequently, the dictionaries are converted into Python list data structures for increased object versatility. Each row within the worksheets is stored in Python list objects embedded in the worksheet list.

2. Control Board

The control board is the operational method section of the code. The control board utilizes user input to define the way data are sorted using a tiered structure (primary, secondary, and table sorting). Each tier receives integer values, which indicate how to sort the data, which data to extract, and how to identify and describe the data in a table accompanying the output visualization. These integer values are set by the user input in the GUI and packaged into a list for subsequent processing.

3. Data Reduction

The data reduction section of the code contains all of the methods and functions necessary to reduce the data for visualization and graphing as per the user request. A collection of methods is used based upon the information gathered from the control board section of the code. For example, if the user requests the SMT name as the primary data sorting and the Capability Area as the secondary data sorting, then the data reduction section of the code reduces the data and orders the data lists first by SMT, followed by Capability Area.

4. Graphing

Once the data have been reduced as per the user input from the control board, the data are ready to be graphed. Using the matplotlib library within Python, the code systematically plots the reduced data, enabling and disabling various elements on the graph based upon user input. For example, the graphing section allows the user to plot the gap-closing tasks associated with a SMT performance gap alongside the gap, if the user opts to do so. The graphing section returns multiple plotted images identified by the page number associated with the reduced data and stores them in a temporary image cache.

5. Data Export

The data export section of the code prepares an output visualization of the data by using the pptx library from within Python. The data export section takes the temporarily cached images, as well as 
various preset images stored within the program directory, and compiles the customized SCOREboard onto a Microsoft PowerPoint Presentation file. This section simultaneously opens PowerPoint so that the customized SCOREboard can be viewed.

\section{C.2. Graphical Code}

The graphical code is GUI based and makes up the second portion of the source code. The graphical code runs the SCOREboard GUI and simultaneously communicates with the functional code during the process. The SCOREboard GUI was developed in Qt Designer, a cross platform developing environment for GUIs. It was written using the PyQt library, which is the Python binding for $Q t$.

The GUI presents two options to the user: 1) a traditional SCOREboard option and 2) a SCOREboard builder option. The traditional SCOREboard option assembles a SCOREboard based upon: primary sort = SMT, secondary sort $=$ capability area, and table sort $=$ testing location. This option offers a quicker route to producing presentations of a standard format, with the ability to produce updated versions as the data repository is updated. The second option, the SCOREboard builder (Figure 7), allows the user to use the tiered sorting system to produce customized SCOREboards based upon the user input.

The GUI serves as both a controller of and a restrictor for the user input. The GUI ensures that the code is being used properly as it restricts the user from requests that are either nonsensical based on the tiered structure or that would result in a operational methods error due to layout and functionality of the program code. Additionally, the GUI assists the user to produce customized SCOREboards quickly and efficiently.

\section{Human Exploration Capability Roadmaps}

The traditional SCOREboard capability roadmap is presented in Figure 9 using notional SMT data as an example. The primary sort is by SMT, the secondary sort is by capability area, and the table is sorted by gap testing location. A gap-level SCOREboard and a task-level SCOREboard are generated for each SMT using this template configuration. Presented here is information on how to interpret the SCOREboards using Figure 9 as an example, followed by discussions on how the collective FY 2015 roadmaps are used to: 1) communicate capability developments and readiness of systems for future missions, and 2) influence the definition of NASA's human exploration investment portfolio through capability-driven processes.

\section{A. How to interpret a SCOREboard}

Each of the tasks underneath the parent gap adds up to the total gap development duration making it visually easy to identify when and in what order the tasks need to be performed in order for the parent gap to be closed with respect to performance. The start date for each gap-closing task is calculated first by subtracting the task duration from the parent gap mission need launch date, relative to the phasing of each task underneath the parent gap. Second, a notional period of time during which preliminary and critical design reviews will be conducted prior to launch is also subtracted from the launch date in order to determine the task start date plotted on the SCOREboard. This notional review period is assumed to be 6 years prior to launch for human missions (cargo and crew) to the Mars system and 3 years for all other missions. This design review period is included in the task start data calculation to ensure that required capability deliverables will be successfully integrated into mission plans.

Each performance gap is mapped to the first notional mission for which the gap is closed and specified within the bracket next to the gap title in the table. Please refer to Figure 3 for a listing of notional missions by phase. Once a gap is closed for a mission, then it is assumed the performance is available for that mission and all subsequent missions. Furthermore, once all the gaps are closed in each capability area, then that entire capability is ready to infuse into Mars vicinity and Mars surface missions.

The SCOREboards are a snapshot in time and represent notional SMT data corresponding to the end of each fiscal year. The notional SMT data repository is updated at the end of each fiscal year at which time new SCOREboards are generated.

\section{B. Capability developments and readiness of systems for future missions}

When viewed together, the collective SCOREboard capability roadmaps tell the story of human exploration capability developments and the subsequent readiness of advanced systems for future missions. 


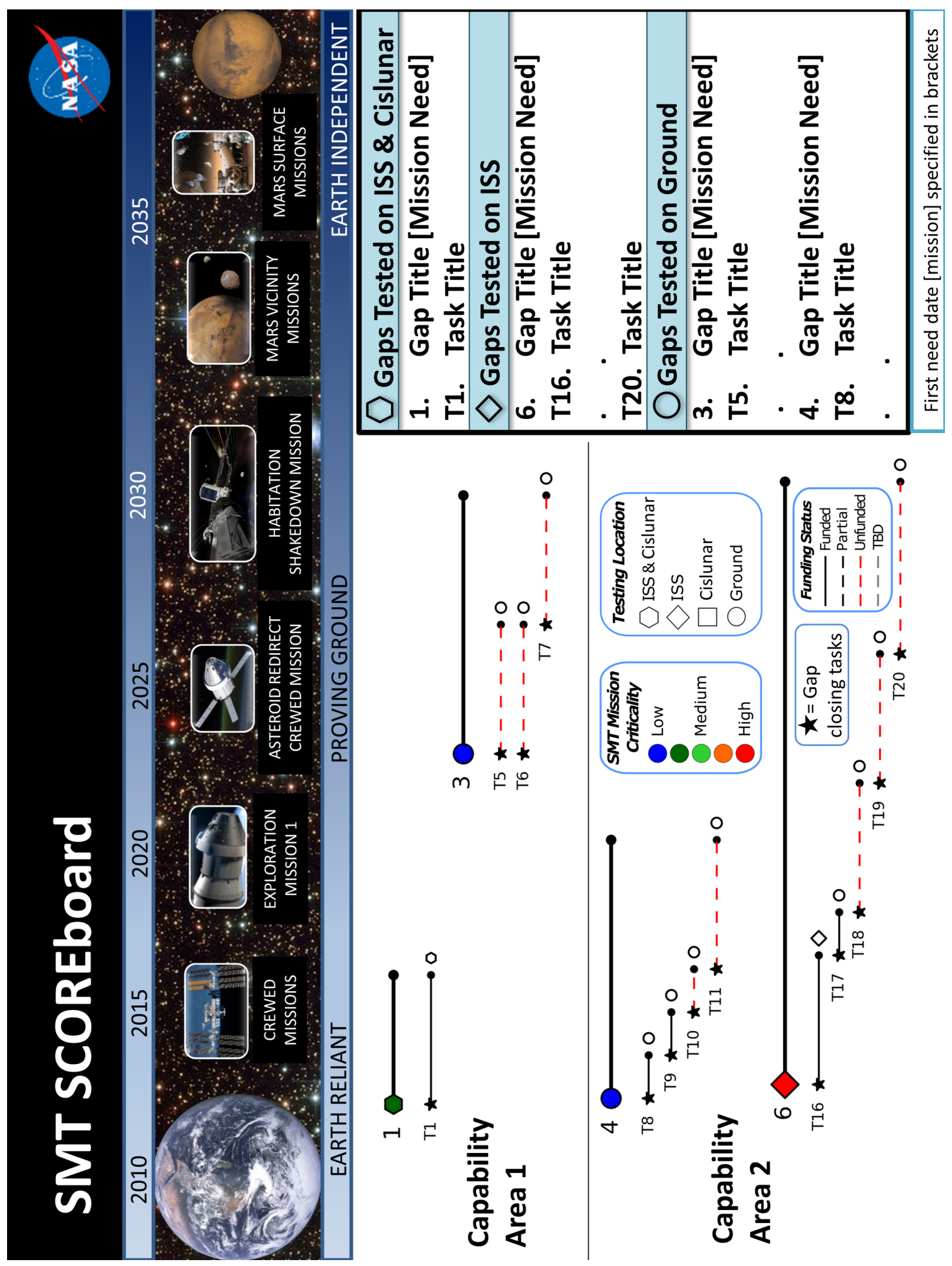

Figure 9. Traditional SCOREboard template using: primary sort = System Maturation Team, secondary sort $=$ capability area, table sort $=$ testing location. 


\section{B.1. Variability of the number of gaps and tasks}

The number of gaps and tasks varies by SMT. This variability is linked to the granularity of the data (Figure 6). SMTs in the high granularity group generally identify more gaps and tasks than those in the mid and low granularity groups because of lessons learned during long term use of their systems in a relevant environment. The average number of both the gaps and tasks is the highest for the high granularity group and is lowest for the low granularity group when excluding the ISRU data. Even though the ISRU data fall within the description of the low granularity group, the ISRU gap list is long because multiple technology solutions are offered for alternate architecture approaches (more discussion to follow in §B.4).

The variability of the number of gaps and tasks per SMT is also affected by how each SMT chooses to group and roll up their data to higher levels. For example, the ECLSS SMT defines numerous tasks to close each gap, while other SMTs such as EVA, roll many activities into a few number of tasks and use just these few tasks to close a parent gap.

Additionally, this variability does not imply that capability areas with more gaps or tasks are more technically difficult to mature than systems and capabilities with fewer gaps or tasks. For example, Gap \#1 in Figure 9 is closed with the completion of one task that requires testing on both the ISS and in cislunar space, while Gap \#4 is closed with the completion of four tasks that require ground testing. An assessment of technical difficulty is not realistic based on comparison of the number of tasks between these two gaps.

\section{B.2. Near-term emphasis}

The SCOREboards highlight a significant workload in the near-term in order to ready systems and mature capabilities for Mars vicinity and Mars surface missions. Every SMT identifies gap-closing tasks to begin prior 2020; work needs to begin on closing $91 \%$ of the SMT-identified capability gaps. Eighty-one percent of the identified gap-closing tasks will require testing by 2020, of which, $51 \%$ need to be tested on ground, $29 \%$ need to be tested on ISS or on an ISS resupply vehicle (Fire Safety), and 17\% need to be tested in cislunar space. These data indicate that numerous parallel development efforts may be required before 2020 .

\section{B.3. Capability developments}

Gap development timelines indicate the time when gap-closing tasks need to begin in order for capabilities to be ready to infuse into a desired notional mission. All tasks are completed and then the performance gap is closed, meaning that the performance for the advanced capability is achieved. Generally, multiple advanced capabilities are required for a single notional mission. As gaps close, the systems attain readiness for notional missions. Readiness for notional missions is determined by: 1) if the gap-closing date occurs before the expected preliminary and critical design review period prior to launch, and 2) if all the gap-closing tasks are fully funded.

The SCOREboard format allows the reader to easily identify the funding status (fully funded, partially funded, or unfunded) of all of the gap-closing tasks. The funding of each performance gap can then be determined. The funding status combined with capability gap development timelines indicate developments that may fall behind schedule. For example, Gap \#4 and Gap \#6 in Figure 9 are currently in progress of closing, but some of their tasks are not funded. This highlights a potential disconnect in the systems planned for the notional mission and warrants further dialog between the system planners (SMTs), the system users (EMC architecture team), and the stakeholders (HEOMD).

\section{B.4. Multiple technology solutions}

Some SMTs identify task development timelines for multiple technology solutions to close a single gap or to achieve the desired performance of an advanced capability. In addition, some SMTs identify multiple technology solutions regarding alternate architecture approaches within the conceptual tradespace of the EMC framework. These options are presented on the SCOREboards because future notional missions are not yet well defined. These multiple technology solutions are used to inform the EMC architecture team and the stakeholders on the benefits and risks associated with each technology option. Once future notional missions become well defined, focused technology solutions will be selected to close the gap or to advance the capability. 


\section{NASA's human exploration investment portfolio}

The SCOREboards show an overview of human exploration capability developments required to enable the J2M through the framework of the EMC. These capability roadmaps are used to influence the definition of NASA's human exploration investment portfolio by illustrating development timelines, testing locations, and funding status of key system maturation activities.

\section{C.1. Influencing through dialog}

The SCOREboards are used to promote dialog between the SMTs, the EMC architecture team, and the stakeholders to make sure that all these groups are on the same page with respect to moving toward common human exploration initiatives. The SMT Integration Team and the SMTs use the SCOREboards to communicate comprehensive human exploration maturation activities to stakeholders. The SMTs also use the SCOREboards to describe which of their performance gaps and tasks require funding to mature capabilities needed for human exploration.

For example, the SCOREboards indicate that numerous parallel development efforts may be required by 2020; however, only $49 \%$ of the gap-closing tasks that require testing by 2020 are fully funded or partially funded. This implies a heavy emphasis on near-term investments may be required. Stakeholders use this information for budget planning purposes and decision making regarding testing opportunity aboard ISS. The collective information on funding status combined with parallel development efforts allows stakeholders to shape the human exploration investment portfolio by prioritizing investments.

The task testing locations presented along with the capability development timelines provide important information regarding ISS and cislunar space utilization. The identified testing locations presented in the SCOREboards are used by EMC element designers and architects to understand how the required system testing may impact element designs and mission cadence at the campaign level. Stakeholders use the testing location data for budget planning and investment prioritization.

\section{C.2. Capability-driven processes}

The SCOREboards also highlight that $67 \%$ of the total gaps need to be closed in support of missions during Phase 1 and Phase 2 of the Proving Ground. Closing performance gaps early on reduces the number of gaps that need to close in support of later Mars vicinity and Mars surface missions. This approach follows the CDF in that each phase focuses on incrementally building, testing, and validating critical capabilities required to eventually field long-duration crewed missions to the Mars system. The majority of SMTs (Comm/Nav, EDL, EVA, Fire Safety, Robotics, ISRU, Power, and SMMP) plan to close 50\% or more of their gaps in support of Phase 1 and Phase 2 missions.

Following the CDF approach provides increased flexibility because once gaps are closed in support of earlier Proving Ground missions, then these capabilities can be infused into any defined mission with little deviation from early planning using notional missions. This approach will open up many potential destinations for human spaceflight throughout the solar system. Additionally, closing numerous gaps in the Proving Ground allows for Mars vicinity and Mars surface mission funding to be spread out throughout the 2020s and the 2030s instead of just in the 2030s when humans will travel to the Mars system.

\section{Summary and Future Work}

SCOREboards are graphical visualizations of SMT capability development needs that enable the EMC architecture and notional missions. SCOREboards present comprehensive overviews of development timelines, testing locations, and funding status that when viewed together provide a roadmap of system maturation needs critical for the J2M. These visualization roadmaps integrate differing data granularity levels through a unified format designed to achieve comparisons among datasets.

Comparisons of SCOREboards provide insight into the following:

- Numerous parallel near-term development efforts may be required to ready systems and mature capabilities for Mars vicinity and Mars surface missions.

- Further dialog is warranted between system planners, system users, and stakeholders when assessing the impact of capability maturation funding status. 
- Multiple technology solutions are used to facilitate dialog regarding alternate capability development timelines and infusion into notional missions.

- Testing locations combined with development timelines are used to understand how required system testing may impact element designs, mission cadence, budget planning, and investment prioritization.

- Closing numerous gaps in the Proving Ground opens up many potential destinations for human spaceflight throughout the solar system.

SCOREboards are generated by a computer program that provides the flexibility not only to plot the traditional format illustrated in Figure 9, but also to plot a variety of formats specified by the user as requested by stakeholders. The GUI facilitates a quick turnaround of SCOREboard data products to address stakeholder needs. Alternate formats include those that emphasize capability developments that are both near-term and the highest mission criticality level, and those that specify developments that require testing on the ISS or in cislunar space.

These alternate formats combined with the traditional SCOREboard format are used to describe cohesive human exploration capability stories that promote valuable dialog between SMTs, EMC element designers and architects, and stakeholders. From this dialog comes an awareness and understanding among the planners, designers, and decision makers that is used to move toward common human exploration initiatives. These initiatives drive NASA's exploration investments within the capability-driven framework.

Recent work on the SCOREboard computer code has focused on including the ability to visualize capability development needs that enable the notional EMC architecture elements instead of the notional missions. When completed, these types of visualizations will present overviews of all desired capability performance required for each element. Notional missions are made up of multiple elements. Therefore, elements can be rearranged to assemble alternate notional missions while at the same time assessing the impact of required capability needs.

Future work involves generating a series of new SCOREboards using FY 2016 SMT capability data. These visualizations will be used to communicate updated capability developments that enable the evolving architecture of the EMC. This iterative process of using SCOREboards to promote dialog between the SMTs and EMC element designers is critical to understanding the advanced capabilities required for the J2M.

Future work also includes making the code extensible to other architectures beyond the EMC. The SCOREboards are impactful visualizations that can be used to communicate capability developments and readiness of systems for any human exploration architecture.

\section{Acknowledgments}

The authors of this paper would like to acknowledge all the SMT leads and team members for the data products used in these analyses.

\section{References}

\footnotetext{
${ }^{1}$ Craig, D., Troutman, P., and Herrmann, N., "Pioneering Space Through an Evolvable Mars Campaign," AIAA SPACE Conference and Exposition, 2015.

${ }^{2}$ Williams-Byrd, J., Arney, D., Rodgers, E., Antol, J., Simon, M., Hay, J., and Larman, K., "Implementing NASAs Capability-Driven Approach: Insight into NASAs Process for Maturing Exploration Systems," AIAA SPACE Conference and Exposition, 2015.

3 "NASA's Journey to Mars: Pioneering Next Steps in Space Exploration," NASA, 2015, http://www.nasa.gov/sites/ default/files/atoms/files/journey-to-mars-next-steps-20151008_508.pdf [Accessed: August 1, 2016].

${ }^{4}$ Goodliff, K., Troutman, P., Craig, D., and Herrmann, N., "Evolvable Mars Campaign 2016 Analysis Update," AIAA SPACE Conference and Exposition, 2016.

${ }^{5}$ Merrill, R., Komar, D., Qu, M., and Chai, P., "Mars Sphere of Influence Maneuvers for NASAs Evolvable Mars Campaign," AIAA SPACE Conference and Exposition, 2016.

${ }^{6}$ Percy, T., Polsgrove, T., Alexander, L., and Turpin, J., "Design and Development of a Methane Cryogenic Propulsion Stage for Human Mars Exploration," AIAA SPACE Conference and Exposition, 2016.
} 